\title{
Recent highlights in antimalarial drug resistance and chemotherapy research
}

\author{
David A. Fidock ${ }^{1,2}$, Richard T. Eastman ${ }^{1}$, Stephen A. Ward ${ }^{3}$, and Steven R. Meshnick ${ }^{4}$ \\ ${ }^{1}$ Department of Microbiology, Columbia University College of Physicians and Surgeons, New York, \\ NY 10032, USA \\ ${ }^{2}$ Department of Medicine, Columbia University College of Physicians and Surgeons, New York, NY \\ 10032, USA \\ ${ }^{3}$ Department of Molecular and Biochemical Parasitology, Liverpool School of Tropical Medicine, \\ Liverpool L3 5QA, UK \\ ${ }^{4}$ Department of Epidemiology, University of North Carolina, Chapel Hill NC 27599, USA
}

\begin{abstract}
This review summarizes recent investigations into antimalarial drug resistance and chemotherapy, including reports of some of the many exciting talks and posters on this topic that were presented at the third Molecular Approaches to Malaria meeting held in Lorne, Australia, in February 2008 (MAM 2008). After surveying this area of research, we focus on two important questions: what is the molecular contribution of $p f c r t$ to chloroquine resistance, and what is the mechanism of action of artemisinin? We conclude with thoughts about the current state of antimalarial chemotherapy and priorities moving forward.
\end{abstract}

\section{The search for new antimalarials}

For decades, malaria chemotherapy has relied on a limited number of drugs. However, the acquisition and spread of drug resistance has led to an increase in morbidity and mortality rates in many malaria-endemic regions. This increasing burden caused by drug-resistant parasites has stimulated investigators to seek out novel antimalarial inhibitors and drug targets, and to define the genetic basis of resistance to existing drugs, as a means to facilitate detection and develop novel strategies to overcome resistance.

\section{Targeting chromatin-modifying enzymes}

The post-genomic era has ushered in a renewed focus on mechanisms of gene regulation in Plasmodium, driven largely by investigations into the process of allelic exclusion in the var gene family [1]. These have revealed a key role for the Plasmodium falciparum histone deacetylase (HDAC) Sir2, the function of which in dynamic chromatin remodeling is intimately associated with var gene silencing [2,3]. Earlier studies had identified HDAC inhibitors (Table 1 ) that had moderate potency against cultured $P$. falciparum parasites in vitro and rodent malaria parasites in vivo (see, for example, Ref. [4]). At MAM 2008, Kathy Andrews (Queensland Institute of Medical Research, Australia) and colleagues reported highly potent in vitro inhibitors, with $\mathrm{IC}_{50}$ values as low as $3 \mathrm{nM}$, which possess high selectivity for $P$. falciparum compared to mammalian cells [5]. These inhibitors hyperacetylate parasite

(c) 2008 Elsevier Ltd. All rights reserved.

Corresponding author: Fidock, D.A. (E-mail: df2260@columbia.edu). 
histones and produce an altered RNA-expression profile for some $P$. falciparum genes. Zbynek Bozdech (Nanyang Technological University, Singapore) reported similar findings with the HDAC inhibitor apicidin (abstracts for these and other studies reported at MAM 2008 appear in a Supplement to the Int. J. Parasitol.) ${ }^{*}$.

\section{The apicoplast - how good a target?}

Additional novel drug targets are thought to reside within the parasite apicoplast, an organelle ancestrally related to chloroplasts [6]. In describing these, Geoff McFadden (University of Melbourne, Australia) discussed the apicoplast type II fatty acid synthesis pathway, which has been postulated to be the target of several microbicides including triclosan and thiolactomycin [6]. Groups led by David Fidock (Columbia University, New York, NY) and Stefan Kappe (Seattle Biomedical Research Institute, Seattle, WA) have recently found that, unexpectedly, this pathway is non-essential in asexual blood stages but is key to the development of infectious liver-stage forms. A more promising apicoplast pathway for the development of an effective blood-stage antimalarial is that of isoprenoid biosynthesis. Proof of principle has been achieved already with the demonstration that fosmidomycin, an inhibitor of the isoprenoid enzyme 1deoxy-D-xylulose 5-phosphate (DOXP) reductase, can clinically cure $P$. falciparum infection either with extended monotherapy or as a component of shorter-duration combination therapies [7]. The challenge is to identify fosmidomycin analogs that have a more suitable pharmacokinetic profile. For now, the most clinically useful apicoplast-specific antimalarials are the antibiotics, such as clindamycin, tetracycline and azithromycin, that target protein translation. These antibacterials, which produce a 'delayed death' phenotype with Plasmodium parasites, have proven to be fairly effective components of antimalarial combinations [6,8]. In vitro resistance to azithromycin, however, can be acquired readily with P. falciparum asexual blood-stage cultures [9]. To make these antibiotic classes more effective as antimalarials will require analog synthesis programs that can identify compounds with greater potency and a reduced propensity to succumb to resistance.

\section{Parasite metabolism}

Inhibitors that arrest parasite metabolism or transport processes are now attracting considerable interest. One area is vitamin biosynthesis, which occurs in $P$. falciparum but is absent in humans. Carsten Wrenger (Bernhard Nocht Institute for Tropical Medicine, Hamburg, Germany) presented the development of prodrugs that upon activation by pyridoxine kinase can inhibit pyridoxal phosphate. Another pathway currently being targeted is the biosynthesis of coenzyme A from the precursor pantothenate (vitamin $\mathrm{B}_{5}$ ). Kevin Saliba and his group (Australian National University, Canberra, Australia) have now identified analogs of pantothenate that possess selective antimalarial activity, with $\mathrm{IC}_{50}$ values of $15 \mu \mathrm{M}$ or higher [10].

\section{Parasite transporters}

Transporters involved in nutrient acquisition from the host are also under investigation as candidate targets. Enrique Salcedo from the group of Patrick Bray (Liverpool School of Tropical Medicine, UK) reported that the parasite-encoded folate-biopterin transporters PfFBT1 and PfFBT2 can be inhibited by the dihydrofolate reductase inhibitor metho-trexate and the organic anion transporter inhibitor probenecid. Folic acid uptake can also be inhibited by the folate precursor para-amino benzoic acid, indicating a wide spectrum of substrates for these two parasite transporters. An association between energy-dependent uptake of folates and mitochondrial processes has also been observed by Ping Wang in the group of John Hyde (University of Manchester, UK), based on studies with compounds known to inhibit

\footnotetext{
*MAM 2008, Lorne, Australia, Int. J. Parasitolol. 38, Suppl. 1, S1-S138.
} 
mitochondrial processes in other systems (see Ref. [11] and data reported at MAM 2008)* Sanjeev Krishna (St. George's Hospital Medical School, London, UK) and Kevin Saliba are also exploring inhibitors of the hexose transporter PfHT $[12,13]$ and the P. falciparum V-type $\mathrm{H}^{+}$-ATPase [14], respectively.

\section{Choline biosynthesis}

Unquestionably, the most advanced antimalarial drug development program targeting parasite metabolism relates to inhibition of de novo phosphatidylcholine biosynthesis (Figure 1). This program, spearheaded by Henri Vial (CNRS and University of Montpellier, France), has identified choline analogs that inhibit $P$. falciparum asexual blood stages at single-digit nanomolar concentrations [15]. Proof of fully curative antimalarial activity with short-course treatments has been obtained in rodents and non-human primates infected at high parasitemias $[16,17]$. These compounds are thought to inhibit choline transport and also exert an effect on parasite phospholipid biosynthesis. These multiple modes of action, distinct from current antimalarial agents, are a major strength of these inhibitors [18] because they could help delay the development of resistance. Human clinical trials with this exciting new class of compounds are currently under way, operated by Sanofi-Aventis (H. Vial, personal communication).

\section{Inhibition of mitochondrial enzymes}

Compounds that inhibit pyrimidine de novo biosynthesis also provide promise for future development. Major efforts in this direction are being applied to dihydroorotate dehydrogenase (DHODH), a key flavin mononucleotide-dependent mitochondrial enzyme that utilizes coenzyme Q as the final electron acceptor [19,20]. Margaret Phillips (UT Southwestern Medical Center, Dallas, TX) and colleagues recently have identified several potent, speciesselective inhibitors of $P f \mathrm{DHODH}$ [21]. These inhibitors display $\mathrm{IC}_{50}$ values in the range 20$600 \mathrm{nM}$ and Exhibit 200-20000-fold selectivity for the malarial over the human enzyme. These completely block the Q-dependent oxidation of flavin mononucleotide, indicating that they act upon the electron transfer pathway between flavin mononucleotide and coenzyme-Q [22]. Giancarlo Biagini and Steve Ward (Liverpool School of Tropical Medicine, Liver-pool, UK) have also identified a new class of mitochondrial inhibitors, known as dihydroacridinediones. These inhibitors target the quinol oxidation site $\left(\mathrm{Q}_{\mathrm{o}}\right)$ of the parasite mitochondrion bc1 complex, causing a collapse of the mitochondrion membrane potential and cell death, and display selectivity against the parasite enzyme that is $\sim 5000$-fold higher than for human liver bc1 [23].

\section{Inhibition of parasite invasion of red blood cells}

Drugs could also potentially be designed that inhibit parasite invasion of host red blood cells (RBCs). One mechanism of parasite invasion involves the microneme protein EBA-175, which recognizes host-cell sialic-acid-containing glycophorin A [24-26]. Using NMR spectroscopic techniques and molecular modeling, Mark von Itzstein and colleagues (Griffith University, Queensland, Australia), are exploring the possibility of using neuraminic acid derivatives to compete for sialic acid recognition by EBA-175. Perturbation of this interaction might decrease or prevent parasite invasion of host RBCs.

\section{Inhibition of parasite proteases}

The falcipain and plasmepsin families of cysteine and aspartic proteases, respectively, which are involved in parasite-mediated hemoglobin digestion in the digestive vacuole (DV) of intraerythrocytic parasites, have long been defined as promising drug targets [27,28]. In collaboration with Philip Rosenthal (University of California, San Francisco, CA), scientists at GlaxoSmithKline (Tres Cantos, Spain) have identified inhibitors of falcipains 2 and 3 that prevent in vitro $P$. falciparum growth at low nanomolar concentrations. These inhibitors can 
also cure $P$. falciparum infections propagated in vivo in human RBCs in a new immunocompromised mouse model [29]. Drug discovery efforts focused on the plasmepsin enzymes have lessened after the discovery of ten plasmepsin paralogs in the P. falciparum genome and the finding that parasites can survive, albeit with a reduced growth rate, without all four plasmepsins of the DV [30,31]. Recent studies by Dan Goldberg (Washington University School of Medicine, St. Louis, MO) now reveal substantial redundancy within and between these two hemoglobinase families and show that the plasmepsin zymogens can be cleaved and activated by falcipains or autoactivated in the presence of falcipain inhibitors [32]. Interestingly, HIV protease inhibitors, which are believed to target one or more parasite aspartic proteases, have been found to be effective against malarial infection. Kathy Andrews and Tina Skinner-Adams (Queensland Institute of Medical Research, Australia) presented their data with HIV protease inhibitors tested against $P$. falciparum in vitro or Plasmodium chabaudi in vivo (reviewed in Ref. [33]). These findings are particularly promising in light of the growing use of these protease inhibitors for the treatment of HIV in malaria-endemic regions.

\section{Emerging technologies}

The ability to identify new drug targets and antimalarial compounds through large-scale screens is rapidly improving with emerging high throughput technologies. Vicky Avery (Griffith University, Queensland, Australia) and colleagues are using a DAPI nuclear dye-based highthroughput confocal imaging assay to screen natural product libraries for whole-cell antimalarial activity and have identified several plant extracts with micromolar activities [34]. Whole-cell assays using fluorescence-activated cell sorting analysis of a green fluorescence protein-expressing $P$. falciparum line are also being developed by Chairat Uthaipibull (National Center for Genetic Engineering and Biotechnology, Bangkok, Thailand) in collaboration with Brendan Crabb (the Burnet Institute, Melbourne, Australia) and Alan Cowman (The Water and Eliza Hall Institute, Melbourne, Australia), as part of the WHO TDRsupported Transfection Network. Elizabeth Winzeler (Genomics Institute of the Novartis Research Foundation and The Scripps Research Institute, La Jolla, CA) and colleagues have also developed a 1536-well, SYBR green I fluorescence-based parasite proliferation assay that was used to screen a Novartis collection of 2.6 million chemically diverse compounds, producing more than 9000 active antimalarial hits [35].

Powerful new 'omic' technologies are also being developed to explore drug mode of action and resistance. A quantitative proteomics approach [36], developed by the group of John Yates III (The Scripps Research Institute, La Jolla, CA), is being used to identify parasite proteins that have expression levels that are altered in response to chloroquine (CQ) or artemisinin treatment. Joe DeRisi (University of California, San Francisco, CA) has also developed highthroughput platforms for selecting drug-resistant $P$. falciparum mutants and is screening for transcriptional or sequence changes using microarrays and Solexa sequencing. Sarah Volkman and Dyann Wirth (Harvard School of Public Health, Boston, MA) are developing an extensive genome-wide map of $P$. falciparum genetic diversity that has identified over 93000 single nucleotide polymorphisms and can detect selective sweeps of drug resistance determinants (such as $p f c r t$ ) and define parasite population structures [37]. This work also produced a molecular barcode, based on 24 PCR primer pairs, which ascribes a unique genotype to any strain. This technology has tremendous potential to identify genes associated with emerging drug resistance.

\section{PfCRT and chloroquine resistance}

Multiple laboratory and clinical studies illustrate the central role that PfCRT has in mediating chloroquine resistance (CQR) [38]. How this is achieved mechanistically, and to what extent other parasite factors contribute to resistance, remains an active area of exploration. CQ accumulates in the acidic parasite DV by virtue of its weak base properties. There, it is thought 
to act by binding to toxic heme species, which form during hemoglobin degradation, and inhibiting their detoxification. The CQR phenotype, which is reversible by verapamil, is associated with decreased uptake of CQ in the DV [39]. Previous transfection experiments have shown that mutant $p f c r t$ alleles can confer CQR to a CQ-sensitive (CQS) strain (tested with GC03) and that resistance is dependent on the presence of the PfCRT K76T mutation $[40,41]$.

How do PfCRT mutations lead to CQR? Kiaran Kirk (Australian National University, Canberra, Australia) summarized evidence indicating that $p f c r t$-mediated CQR is a result of increased drug efflux from the DV, by mechanisms that evoke either a carrier or a channel [42-45]. Bioinformatic comparisons with members of the drug-metabolite transporter superfamily [46] and studies in Xenopus laevis oocytes expressing PfCRT indicate that the loss of the positive charge resulting from the K76T mutation enables the protonated drug to traverse the transporter, leading to the movement of drug back into the parasite cytoplasm. This drug translocation is sensitive to verapamil. When the proton pump of the parasite is inhibited by concanamycin A, CQ causes a verapamil-sensitive increase in the rate of DV alkalinization of CQR strains, indicating that CQ efflux is associated with protons [47] (Figure 2).

However, PfCRT mutations are not the full story. Using a transfection-based approach, Stephanie Valderramos and David Fidock (Columbia University, New York) recently have obtained definitive evidence that the genetic background of the host strain determines the extent to which mutant $p f c r t$ exerts $C Q R$. This finding confirms earlier indications obtained with genetically diverse field isolates or culture-adapted lines (reviewed in Ref. [48]). The most unusual response was observed with the transfected D10 clone engineered to express mutant $p f c r t$, in that its decreased susceptibility to $\mathrm{CQ}$ was most pronounced at the $\mathrm{IC}_{90}$ level (i.e. the concentration of drug that inhibits $90 \%$ of growth relative to untreated control) and not the $\mathrm{IC}_{50}$ level. Further experiments revealed that depending on the strain, mutant pfcrt confers either resistance or a state of tolerance, implying that other factors must contribute to enable certain strains to acquire high-level resistance in the presence of mutant $p f c r t$.

Point mutations or changes in the levels of expression of the $p f m d r 1$ gene product Pgh1 can also affect the $P$. falciparum CQR phenotype in a strain-dependent manner [49-52]. Recent studies by David Johnson and Steve Ward have provided evidence that Pgh1 expression can be increased upon parasite exposure to the anticonvulsant phenobarbitone (Figure 2), which causes decreased in vitro CQ susceptibility in both CQR and CQS parasites [53]. This effect is distinct from other studies in which Pgh1 overexpression has been associated with increased CQ susceptibility [54]. In the Johnson study, putative nuclear-receptor-binding sites with homology to mammalian sequences activated by phenobarbitone were identified in the $p f m d r l$ and $p f c r t$ promoter regions, and several putative nuclear receptor genes were identified. These findings raise the novel hypothesis that nuclear-receptor-mediated responses to drug exposure might be a mechanism of gene regulation in P. falciparum [53].

Whereas CQ-resistant strains of $P$. falciparum have spread throughout virtually all malariaendemic areas, CQR in Plasmodium vivax remains relatively rare and is restricted mostly to Indonesia, Irian Jaya and other countries in the Pacific region. The genetic basis of $P$. vivax $\mathrm{CQR}$ has remained elusive and does not seem to involve pvcg10, the $P$. vivax ortholog of pfcrt [55]. Simon Kang'a and others in the group of Jane Carlton (New York University Medical Center, New York, NY) recently surveyed variation across 36 microsatellite markers in 19 CQ-resistant and 21 CQS P. vivax samples from patients in Indonesia. Their preliminary analysis found no evidence of a selective sweep in the resistant isolates and revealed a low level of genetic diversity overall. Ric Price (Menzies School of Health Research, Darwin, Australia) adopted an alternative approach that employed an optimized $P$. vivax in vitro schizont maturation assay applied to $P$. vivax isolates from Papua and Thailand. Genotyping 
analysis of pvmdrl revealed an association between CQR and a novel Y976F mutation [56]. Their data also supported an association between $p v m d r l$ amplification and reduced mefloquine susceptibility, reminiscent of the well-established association between $p f m d r l$ amplification and reduced mefloquine susceptibility [57]. Kasia Modrzynska (University of Edinburgh, UK) has also been pursuing genetic linkage studies with CQ-resistant lines of $P$. chabaudi. These studies found no association with the orthologs of $p f c r t$ or $p f m d r l$ and resulted in the unexpected finding that mutations in the deubiquitinating enzyme UBP-1 occurred in parasites pressured extensively with chloroquine (or artesunate) [58]. Defining the alternative mechanisms of CQR that prevail in other Plasmodium species will be important for a more complete understanding of how resistance to this drug is acquired and mediated.

Many questions remain about CQR and CQ treatment failures in humans. Johan Ursing (Karolinska Institute, Stockholm, Sweden) recently reported findings from Guinea-Bissau that $\mathrm{CQ}$, at double doses, was highly effective - curing $78 \%$ of uncomplicated malaria patients harboring pfcrt K76T genotypes [59]. This raises the question of the relative importance of pharmacokinetics vs genetics as causes of drug failure. Questions about the relationship between fitness costs imposed by mutant $p f c r t$, the dynamics of mixed infections and the level of local CQ use have also been raised recently by Qin Cheng (Australian Army Malaria Institute, Enoggera, Queensland), who found that mutant $p f c r t$ was decreasing in prevalence far more slowly in Hainan Island, China [60] than in Malawi [61] after the cessation of CQ use to treat $P$. falciparum malaria. Importantly, the returning CQS parasites have wild-type $p f c r t$ and are genetically diverse.

Identification of the PfCRT K76T mutation by PCR has been used widely to identify CQR and has been proven to be a highly sensitive marker [62]. However, results need to be interpreted with caution, especially in high-transmission settings, where individual patients tend to be infected with many genetically distinct $P$. falciparum variants. Steve Meshnick (University of North Carolina, Chapel Hill, NC) has applied heteroduplex tracking assays (HTAs) to describe this internal heterogeneity. HTAs can be used to quantify variants and detect minority variants that represent as little as $1 \%$ of the population in a single host, in contrast to classical PCR methods that can often be insensitive below 10-20\%. Using HTA, his group was able to detect the PfCRT K76T-bearing minority variants in $\sim 25 \%$ of Malawian patients - all of whom seemed (using classical PCR) to be infected only with wild-type parasites. HTA was also used to detect rare instances of the DHFR I164L mutation in P. falciparum infections in Malawi [63].

\section{The mechanism of action of artemisinin}

Artemisinin derivatives are the most important newclass of antimalarials and are in widespread use. Studies into the cytotoxic effects of artemisinin derivatives have indicated that their activity might be propagated by interactions between the endoperoxide bridge of the drug and heme-iron, produced during hemoglobin degradation inside the DV. This iron has been proposed to generate free radicals that alkylate and oxidize proteins and lipids within infected RBCs (see Ref. [64] and references therein). This hypothesis is consistent with the finding that artemisinin activity can be potentiated by oxygen and oxidizing agents and attenuated by reducing agents [65]. However, this has been questioned by other studies. One proposed that artemisinins are activated via reductive cleavage of the peroxide bond by intracellular ironsulfur redox centers, which are common to Plasmodium enzymes, and that alkylation of these enzymes could result in parasite death [66]. Interestingly, incubation of parasite lysates with radiolabeled artemisinin identified several interacting proteins, indicating that parasite death might result fromthe alkylation and inactivation of parasite proteins [67,68]. Additional support against the heme hypothesis is that artemisinins are effective against ring-stage parasites that do not seem to have high concentrations of heme [69]. Specific proteins that have been 
proposed to be the target of artemisinins include cysteine proteases [70], proteins of the electron transport chain [71], translationally controlled tumor protein [68] and PfATP6, a SERCA-type $\mathrm{Ca}^{2+}$ ATPase [72].

PfATP6 has received the most attention because its activity in transfected $X$. laevis oocytes is abolished by artemisinin but is unaffected by other antimalarials. This includes the inactive compound desoxyartemisinin, which lacks the endoperoxide bridge [72]. Additional studies in X. laevis have reported that an L263E point mutation can abolish inhibition by artemisinins [73]. Sanjeev Krishna summarized evidence from field studies and heterologous expression systems that support a role for PfATP6 mutations in emerging artemisinin resistance [74]. To test the role of the L263E mutation in parasite response to artemisinins, allelic exchange experiments were carried out in the Fidock laboratory, in collaboration between the two groups. The presentation included Krishna's statistical analyses of IC $_{50}$ values, which showed a skewed distribution of artemisinin and dihydroartemisinin responses in the mutant recombinant parasites expressing the $\mathrm{L} 263 \mathrm{E}$ mutation, with some assays showing higher $\mathrm{IC}_{50}$ values in the L263E mutants when the data were normalized to wild-type controls. The analysis of these data is ongoing and the results are keenly awaited because the role of PfATP6 in artemisinin susceptibility remains a topic of considerable debate.

Additional work investigating the mechanism of action of artemisinins was presented by Maria del Pilar Crespo, working in the group of Leann Tilley (La Trobe University, Melbourne, Australia), who provided evidence that artemisinin derivatives cause early disruption of the parasite DV and do not seem to affect the structure of the endoplasmic reticulum (where PfATP6 is presumably located). Using a proteomic approach, Sumalee Kamchonwongpaisan (National Centre for Genetic Engineering and Biotechnology, Bangkok, Thailand) presented evidence that artemisinins might affect parasite endocytosis of host proteins. Whether artemisinin accumulation in the DV relates to its mode of action, and how PfATP6 might contribute to artemisinin action and potential gain of resistance by Plasmodium parasites, clearly requires further studies.

\section{Concluding remarks}

The increasing awareness of the problems posed by multi-drug-resistant malaria, and of the need to understand the basis of resistance and develop new chemotherapeutic strategies and drugs, has spurred a rapid increase in the volume of research being applied to this area. The topics cited previously illustrate the tremendous gains that can be achieved through the application of genetic, genomic, proteomic, biochemical, synthetic or medicinal chemistry, or structural biology approaches. Today's optimism that artemisinin-based combination therapies provide a sustainable answer as to how to reduce malaria morbidity and mortality finds parallels in earlier campaigns founded on CQ, the efficacy of which was once absolute. The elaboration of a coordinated Worldwide Antimalarial Drug Resistance Network to identify emerging resistance, spearheaded by Carol Sibley (University of Washington, Seattle, WA), is an important initiative in this regard [75]. Ever more research in the area of antimalarial resistance mechanisms and new chemotherapies must be supported to sustain the continuous stream of discoveries needed to reduce the devastating impact of malaria across the inter-tropical regions of the world.

\section{Acknowledgements}

We thank Celeste Diamond Li for excellent help with the preparation of this review and Henri Vial for providing Figure 1. We extend our thanks to the many who helped with the preparation of this review and our apologies to those whose work we could not cite, for reasons of brevity. 


\section{References}

1. Scherf A, et al. SnapShot: var gene expression in the malaria parasite. Cell 2008;134:190. [PubMed: 18614021]

2. Duraisingh MT, et al. Heterochromatin silencing and locus repositioning linked to regulation of virulence genes in Plasmodium falciparum. Cell 2005;121:13-24. [PubMed: 15820675]

3. Freitas-Junior LH, et al. Telomeric heterochromatin propagation and histone acetylation control mutually exclusive expression of antigenic variation genes in malaria parasites. Cell 2005;121:25-36. [PubMed: 15820676]

4. Andrews KT, et al. Anti-malarial effect of histone deacetylation inhibitors and mammalian tumour cytodifferentiating agents. Int. J. Parasitol 2000;30:761-768. [PubMed: 10856511]

5. Andrews KT, et al. Potent antimalarial activity of histone deacetylase inhibitor analogues. Antimicrob. Agents Chemother 2008;52:1454-1461. [PubMed: 18212103]

6. Ralph SA, et al. Tropical infectious diseases: metabolic maps and functions of the Plasmodium falciparum apicoplast. Nat. Rev. Microbiol 2004;2:203-216. [PubMed: 15083156]

7. Wiesner J, Jomaa H. Isoprenoid biosynthesis of the apicoplast as drug target. Curr. Drug Targets 2007;8:3-13. [PubMed: 17266527]

8. Dahl EL, Rosenthal PJ. Apicoplast translation, transcription and genome replication: targets for antimalarial antibiotics. Trends Parasitol 2008;24:279-284. [PubMed: 18450512]

9. Sidhu $\mathrm{AB}$, et al. In vitro efficacy, resistance selection, and structural modeling studies implicate the malarial parasite apicoplast as the target of azithromycin. J. Biol. Chem 2007;282:2494-2504. [PubMed: 17110371]

10. Spry C, et al. A class of pantothenic acid analogs inhibits Plasmodium falciparum pantothenate kinase and represses the proliferation of malaria parasites. Antimicrob. Agents Chemother 2005;49:46494657. [PubMed: 16251308]

11. Wang P, et al. Characterisation of exogenous folate transport in Plasmodium falciparum. Mol. Biochem. Parasitol 2007;154:40-51. [PubMed: 17509698]

12. Joet T, et al. Validation of the hexose transporter of Plasmodium falciparum as a novel drug target. Proc. Natl. Acad. Sci. U. S. A 2003;100:7476-7479. [PubMed: 12792024]

13. Patel AP, et al. New antimalarial targets: the example of glucose transport. Travel Med. Infect. Dis 2008;6:58-66. [PubMed: 18342277]

14. Saliba KJ, et al. Acidification of the malaria parasite's digestive vacuole by $\mathrm{aH}^{+}-\mathrm{ATPase}$ and $\mathrm{aH}^{+}$pyrophosphatase. J. Biol. Chem 2003;278:5605-5612. [PubMed: 12427765]

15. Hamze A, et al. Mono- and bis-thiazolium salts have potent antimalarial activity. J. Med. Chem 2005;48:3639-3643. [PubMed: 15887971]

16. Vial HJ, et al. Prodrugs of bisthiazolium salts are orally potent antimalarials. Proc. Natl. Acad. Sci. U. S. A 2004;101:15458-15463. [PubMed: 15492221]

17. Wengelnik K, et al. A class of potent antimalarials and their specific accumulation in infected erythrocytes. Science 2002;295:1311-1314. [PubMed: 11847346]

18. Richier E, et al. Potent antihematozoan activity of novel bisthiazolium drug T16: evidence for inhibition of phosphatidylcholine metabolism in erythrocytes infected with Babesia and Plasmodium spp. Antimicrob. Agents Chemother 2006;50:3381-3388. [PubMed: 17005821]

19. Baldwin J, et al. Malarial dihydroorotate dehydrogenase. Substrate and inhibitor specificity. J. Biol. Chem 2002;277:41827-41834. [PubMed: 12189151]

20. Painter HJ, et al. Specific role of mitochondrial electron transport in blood-stage Plasmodium falciparum. Nature 2007;446:88-91. [PubMed: 17330044]

21. Phillips MA, et al. Triazolopyrimidine-based dihydroorotate dehydrogenase inhibitors with potent and selective activity against the malaria parasite Plasmodium falciparum. J. Med. Chem 2008;51:3649-3653. [PubMed: 18522386]

22. Malmquist NA, et al. Analysis of flavin oxidation and electron-transfer inhibition in Plasmodium falciparum dihydroorotate dehydrogenase. Biochemistry 2008;47:2466-2475. [PubMed: 18225919]

23. Biagini GA, et al. Acridinediones: selective and potent inhibitors of the malaria parasite mitochondrial bc1 complex. Mol. Pharmacol 2008;73:1347-1355. [PubMed: 18319379] 
24. Duraisingh MT, et al. Erythrocyte-binding antigen 175 mediates invasion in Plasmodium falciparum utilizing sialic acid-dependent and -independent pathways. Proc. Natl. Acad. Sci. U. S. A 2003;100:4796-4801. [PubMed: 12672957]

25. Sim BK, et al. Receptor and ligand domains for invasion of erythrocytes by Plasmodium falciparum. Science 1994;264:1941-1944. [PubMed: 8009226]

26. von Itzstein M, et al. Hot, sweet and sticky: the glycobiology of Plasmodium falciparum. Trends Parasitol 2008;24:210-218. [PubMed: 18420458]

27. Rosenthal PJ. Cysteine proteases of malaria parasites. Int. J. Parasitol 2004;34:1489-1499. [PubMed: 15582526]

28. Goldberg DE. Hemoglobin degradation. Curr. Top. Microbiol. Immunol 2005;295:275-291. [PubMed: 16265895]

29. Angulo-Barturen I, et al. A murine model of falciparum-malaria by in vivo selection of competent strains in non-myelodepleted mice engrafted with human erythrocytes. PLoS One 2008;3:e2252. [PubMed: 18493601]

30. Bonilla JA, et al. Critical roles for the digestive vacuole plasmepsins of Plasmodium falciparum in vacuolar function. Mol. Microbiol 2007;65:64-75. [PubMed: 17581121]

31. Liu J, et al. Plasmodium falciparum ensures its amino acid supply with multiple acquisition pathways and redundant proteolytic enzyme systems. Proc. Natl. Acad. Sci. U. S. A 2006;103:8840-8845. [PubMed: 16731623]

32. Drew ME, et al. Plasmodium food vacuole plasmepsins are activated by falcipains. J. Biol. Chem 2008;283:12870-12876. [PubMed: 18308731]

33. Andrews KT, et al. HIV-malaria interactions: don't forget the drugs. Science 2007;315:1791. [PubMed: 17395812]

34. Fernandez LS, et al. Antimalarial activity of natural product extracts from Papua New Guinean and Australian plants against Plasmodium falciparum. Phytother. Res 2008;22:1409-1412. [PubMed: 18693292]

35. Plouffe D, et al. In silico activity profiling reveals the mechanism of action of antimalarials discovered in a high-throughput screen. Proc. Natl. Acad. Sci. U. S. A 2008;105:9059-9064. [PubMed: 18579783]

36. Washburn MP, et al. Analysis of quantitative proteomic data generated via multidimensional protein identification technology. Anal. Chem 2002;74:1650-1657. [PubMed: 12043600]

37. Volkman SK, et al. A genome-wide map of diversity in Plasmodium falciparum. Nat. Genet 2007;39:113-119. [PubMed: 17159979]

38. Valderramos SG, Fidock DA. Transporters involved in resistance to antimalarial drugs. Trends Pharmacol. Sci 2006;27:594-601. [PubMed: 16996622]

39. Bray PG, et al. Defining the role of PfCRT in P. falciparum chloroquine resistance. Mol. Microbiol 2005;56:323-333. [PubMed: 15813727]

40. Sidhu ABS, et al. Chloroquine resistance in Plasmodium falciparum malaria parasites conferred by pfcrt mutations. Science 2002;298:210-213. [PubMed: 12364805]

41. Lakshmanan V, et al. A critical role for PfCRT K76T in Plasmodium falciparum verapamil-reversible chloroquine resistance. EMBO J 2005;24:2294-2305. [PubMed: 15944738]

42. Bray PG, et al. PfCRT and the trans-vacuolar proton electrochemical gradient: regulating the access of chloroquine to ferriprotoporphyrin IX. Mol. Microbiol 2006;62:238-251. [PubMed: 16956382]

43. Hayward R, et al. The $\mathrm{pH}$ of the digestive vacuole of Plasmodium falciparum is not associated with chloroquine resistance. J. Cell Sci 2006;119:1016-1025. [PubMed: 16492710]

44. Sanchez CP, et al. Differences in trans-stimulated chloroquine efflux kinetics are linked to PfCRT in Plasmodium falciparum. Mol. Microbiol 2007;64:407-420. [PubMed: 17493125]

45. Klonis N, et al. Evaluation of $\mathrm{pH}$ during cytostomal endocytosis and vacuolar catabolism of haemoglobin in Plasmodium falciparum. Biochem. J 2007;407:343-354. [PubMed: 17696875]

46. Martin RE, Kirk K. The malaria parasite's chloroquine resistance transporter is a member of the drug/ metabolite transporter superfamily. Mol. Biol. Evol 2004;21:1938-1949. [PubMed: 15240840]

47. Lehane AM, et al. A verapamil-sensitive chloroquine-associated $\mathrm{H}^{+}$leak from the digestive vacuole in chloroquine-resistant malaria parasites. J. Cell Sci 2008;121:1624-1632. [PubMed: 18445688] 
48. Cooper RA, et al. pfcrt is more than the Plasmodium falciparum chloroquine resistance gene: a functional and evolutionary perspective. Acta Trop 2005;94:170-180. [PubMed: 15866507]

49. Barnes DA, et al. Selection for high-level chloroquine resistance results in deamplification of the pfmdrl gene and increased sensitivity to mefloquine in Plasmodium falciparum. EMBO J 1992;11:3067-3075. [PubMed: 1353446]

50. Reed MB, et al. Targeted disruption of an erythrocyte binding antigen in Plasmodiumfalciparum is associated with a switch toward a sialic acid-independent pathway of invasion. Proc. Natl. Acad. Sci. U. S. A 2000;97:7509-7514. [PubMed: 10861015]

51. Sidhu ABS, et al. pfmdrl mutations contribute to quinine resistance and enhance mefloquine and artemisinin sensitivity in Plasmodium falciparum. Mol. Microbiol 2005;57:913-926. [PubMed: 16091034]

52. Sidhu ABS, et al. Decreasing pfmdrl copy number in Plasmodium falciparum malaria heightens susceptibility to mefloquine, lumefantrine, halofantrine, quinine, and artemisinin. J. Infect. Dis 2006;194:528-535. [PubMed: 16845638]

53. Johnson DJ, et al. Drug-regulated expression of Plasmodium falciparum P-glycoprotein homologue 1: a putative role for nuclear receptors. Antimicrob. Agents Chemother 2008;52:1438-1445. [PubMed: 18195056]

54. Duraisingh MT, Cowman AF. Contribution of the $p f m d r 1$ gene to antimalarial drug-resistance. Acta Trop 2005;94:181-190. [PubMed: 15876420]

55. Nomura T, et al. Evidence for different mechanisms of chloroquine resistance in 2 Plasmodium species that cause human malaria. J. Infect. Dis 2001;183:1653-1661. [PubMed: 11343215]

56. Russell B, et al. Determinants of in vitro drug susceptibility testing of Plasmodium vivax. Antimicrob. Agents Chemother 2008;52:1040-1045. [PubMed: 18180357]

57. Price RN, et al. Mefloquine resistance in Plasmodium falciparum and increased pfmdrl gene copy number. Lancet 2004;364:438-447. [PubMed: 15288742]

58. Hunt $\mathrm{P}$, et al. Gene encoding a de-ubiquitinating enzyme is mutated in artemisinin- and chloroquineresistant rodent malaria parasites. Mol. Microbiol 2007;65:27-40. [PubMed: 17581118]

59. Ursing J, et al. Plasmodium falciparum genotypes associated with chloroquine and amodiaquine resistance in Guinea-Bissau. Am. J. Trop. Med. Hyg 2007;76:844-848. [PubMed: 17488902]

60. Chen N, et al. No genetic bottleneck in Plasmodium falciparum wild-type pfcrt alleles reemerging in Hainan Island, China, following high-level chloroquine resistance. Antimicrob. Agents Chemother 2008;52:345-347. [PubMed: 17954685]

61. Laufer MK, et al. Return of chloroquine antimalarial efficacy in Malawi. N. Engl. J. Med 2006;355:1959-1966. [PubMed: 17093247]

62. Wellems TE, Plowe CV. Chloroquine-resistant malaria. J. Infect. Dis 2001;184:770-776. [PubMed: 11517439]

63. Juliano JJ, et al. Detection of the dihydrofolate reductase-164L mutation in Plasmodium falciparum infections from Malawi by heteroduplex tracking assay. Am. J. Trop. Med. Hyg 2008;78:892-894. [PubMed: 18541765]

64. Kannan R, et al. Reaction of artemisinin with haemoglobin: implications for antimalarial activity. Biochem. J 2005;385:409-418. [PubMed: 15361062]

65. Krungkrai SR, Yuthavong Y. The antimalarial action on Plasmodium falciparum of qinghaosu and artesunate in combination with agents which modulate oxidant stress. Trans. R. Soc. Trop. Med. Hyg 1987;81:710-714. [PubMed: 3329778]

66. Wu Y. How might qinghaosu (artemisinin) and related compounds kill the intraerythrocytic malaria parasite? A chemist's view. Acc. Chem. Res 2002;35:255-259. [PubMed: 12020162]

67. Bhisutthibhan J, Meshnick SR. Immunoprecipitation of $\left[{ }^{3} \mathrm{H}\right]$ dihydroartemisinin translationally controlled tumor protein (TCTP) adducts from Plasmodium falciparum-infected erythrocytes by using anti-TCTP antibodies. Antimicrob. Agents Chemother 2001;45:2397-2399. [PubMed: 11451708]

68. Bhisutthibhan J, et al. The Plasmodium falciparum translationally controlled tumor protein homolog and its reaction with the antimalarial drug artemisinin. J. Biol. Chem 1998;273:16192-16198. [PubMed: 9632675] 
69. Olliaro PL, et al. Possible modes of action of the artemisinintype compounds. Trends Parasitol 2001;17:122-126. [PubMed: 11286794]

70. Pandey AV, et al. Artemisinin, an endoperoxide antimalarial, disrupts the hemoglobin catabolism and heme detoxification systems in malarial parasite. J. Biol. Chem 1999;274:19383-19388. [PubMed: 10383451]

71. Li W, et al. Yeast model uncovers dual roles of mitochondria in action of artemisinin. PLoS Genet 2005;1:e36. [PubMed: 16170412]

72. Eckstein-Ludwig U, et al. Artemisinins target the SERCA of Plasmodium falciparum. Nature 2003;424:957-961. [PubMed: 12931192]

73. Uhlemann AC, et al. A single amino acid residue can determine sensitivity of SERCAs to artemisinins. Nat. Struct. Mol. Biol 2005;12:628-629. [PubMed: 15937493]

74. Krishna S, et al. Re-evaluation of how artemisinins work in light of emerging evidence of in vitro resistance. Trends Mol. Med 2006;12:200-205. [PubMed: 16616639]

75. Sibley $\mathrm{CH}$, et al. A network to monitor antimalarial drug resistance: a plan for moving forward. Trends Parasitol 2008;24:43-48. [PubMed: 18042432]

76. Jana S, Paliwal J. Novel molecular targets for antimalarial chemotherapy. Int. J. Antimicrob. Agents 2007;30:4-10. [PubMed: 17339102]

77. Spry C, et al. Coenzyme A biosynthesis: an antimicrobial drug target. FEMS Microbiol. Rev 2008;32:56-106. [PubMed: 18173393]

78. Skinner-Adams TS, et al. Identification of phosphinate dipeptide analog inhibitors directed against the Plasmodium falciparum M17 leucine aminopeptidase as lead antimalarial compounds. J. Med. Chem 2007;50:6024-6031. [PubMed: 17960925]

79. Chen X, et al. Inhibitors of Plasmodium falciparum methionine aminopeptidase $1 \mathrm{~b}$ possess antimalarial activity. Proc. Natl. Acad. Sci. U. S. A 2006;103:14548-14553. [PubMed: 16983082]

80. Eastman RT, et al. Thematic review series: lipid posttranslational modifications. Fighting parasitic disease by blocking protein farnesylation. J. Lipid Res 2006;47:233-240. [PubMed: 16339110]

81. Gelb MH, et al. Protein farnesyl and N-myristoyl transferases: piggy-back medicinal chemistry targets for the development of antitrypanosomatid and antimalarial therapeutics. Mol. Biochem. Parasitol 2003;126:155-163. [PubMed: 12615314]

82. Jiang L, et al. Potent and selective activity of a combination of thymidine and 1843U89, a folatebased thymidylate synthase inhibitor, against Plasmodium falciparum. Antimicrob. Agents Chemother 2000;44:1047-1050. [PubMed: 10722510]

83. Amadasi A, et al. Pyridoxal 5'e enzymes as targets for therapeutic agents. Curr. Med. Chem 2007;14:1291-1324. [PubMed: 17504214]

84. Blackman MJ. Malarial proteases and host cell egress: an 'emerging' cascade. Cell. Microbiol 2008;10:1925-1934. [PubMed: 18503638]

85. Kumar $S$, et al. Antimalarial drugs inhibiting hemozoin ( $\beta$-hematin) formation: a mechanistic update. Life Sci 2007;80:813-828. [PubMed: 17157328]

86. Nzila A, et al. Chemosensitization of Plasmodium falciparum by probenecid in vitro. Antimicrob. Agents Chemother 2003;47:2108-2112. [PubMed: 12821454]

87. Yanow SK, et al. Potent antimalarial and transmission-blocking activities of centanamycin, a novel DNA-binding agent. J. Infect. Dis 2008;197:527-534. [PubMed: 18275274]

88. Cui L, Miao J. Cytotoxic effect of curcumin on malaria parasite Plasmodium falciparum: inhibition of histone acetylation and generation of reactive oxygen species. Antimicrob. Agents Chemother 2007;51:488-494. [PubMed: 17145789]

89. Murphy SC, et al. Erythrocyte G protein as a novel target for malarial chemotherapy. PLoS Med 2006;3:e528. [PubMed: 17194200] 


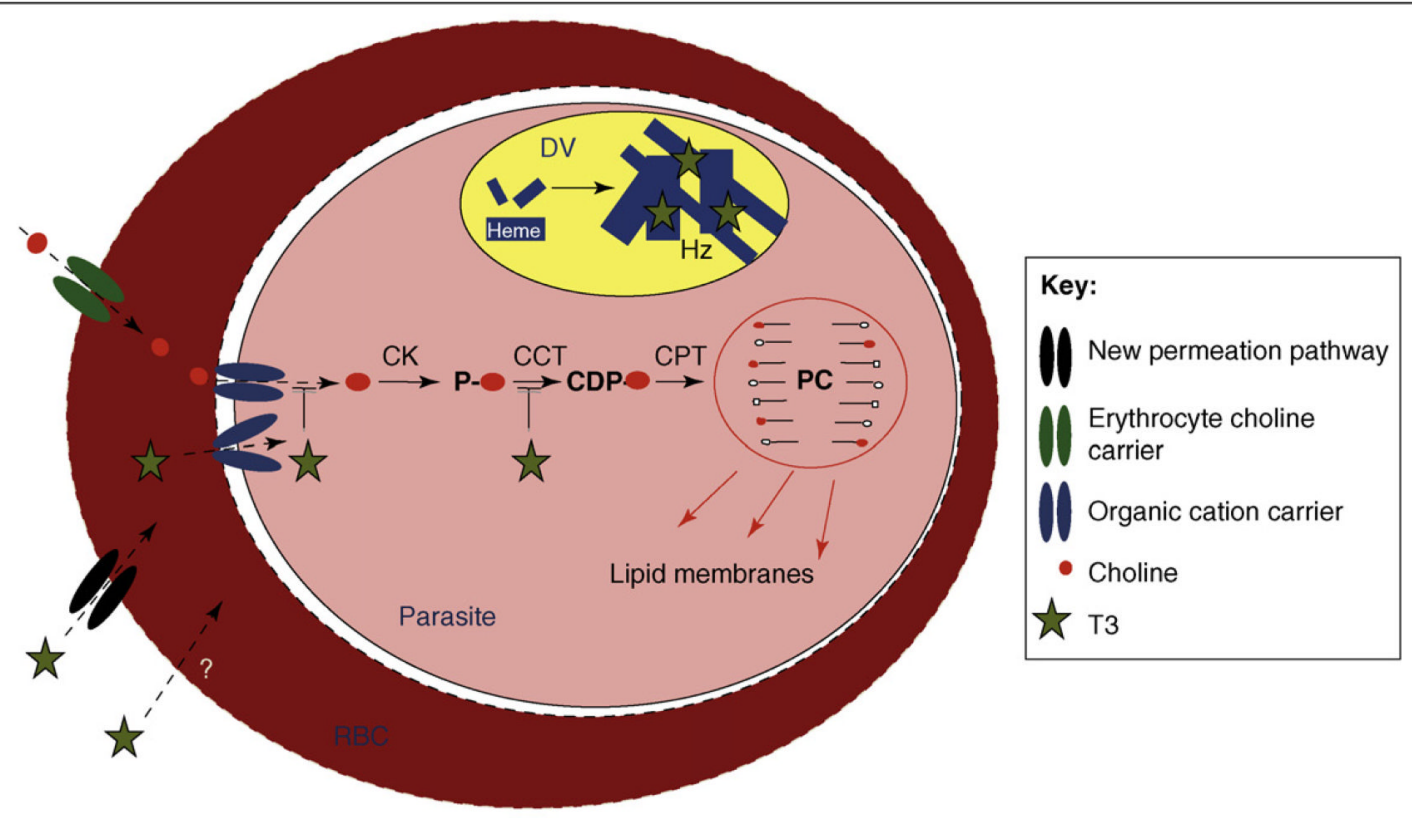

$\overline{\text { TRENDS in Parasitology }}$

Figure 1.

Mechanism of the antimalarial activity of the choline analog T3. This representative bisquaternary ammonium compound is thought to enter the infected erythrocyte through the new permeation pathway or another (unknown) mechanism (represented by the question mark near the RBC membrane). This choline analog is then taken up into the intraerythrocytic parasite via an organic cation transporter present on the parasite plasma membrane. This perturbs choline uptake from the host cell compartment, thus disrupting the synthesis of the predominant membrane lipid, phosphatidylcholine. Additional effects on phosphatidylcholine biosynthesis enzymes have also been proposed [16]. T3 and other biscationic compounds also accumulate within the parasite, mostly in the digestive vacuole (DV, yellow) where they bind heme (ferriprotoporphyrin IX). This heme interaction has also been hypothesized to contribute to their antiplasmodial activity [18]. These compounds, therefore, might have multiple sites of action within the parasite. CCT, phosphocholine cytidylyltransferase; CDP, cytidine diphosphate; CK, choline kinase; CPT, choline phosphate transferase; Hz, hemozoin; P, phosphate; RBC, red blood cell. 


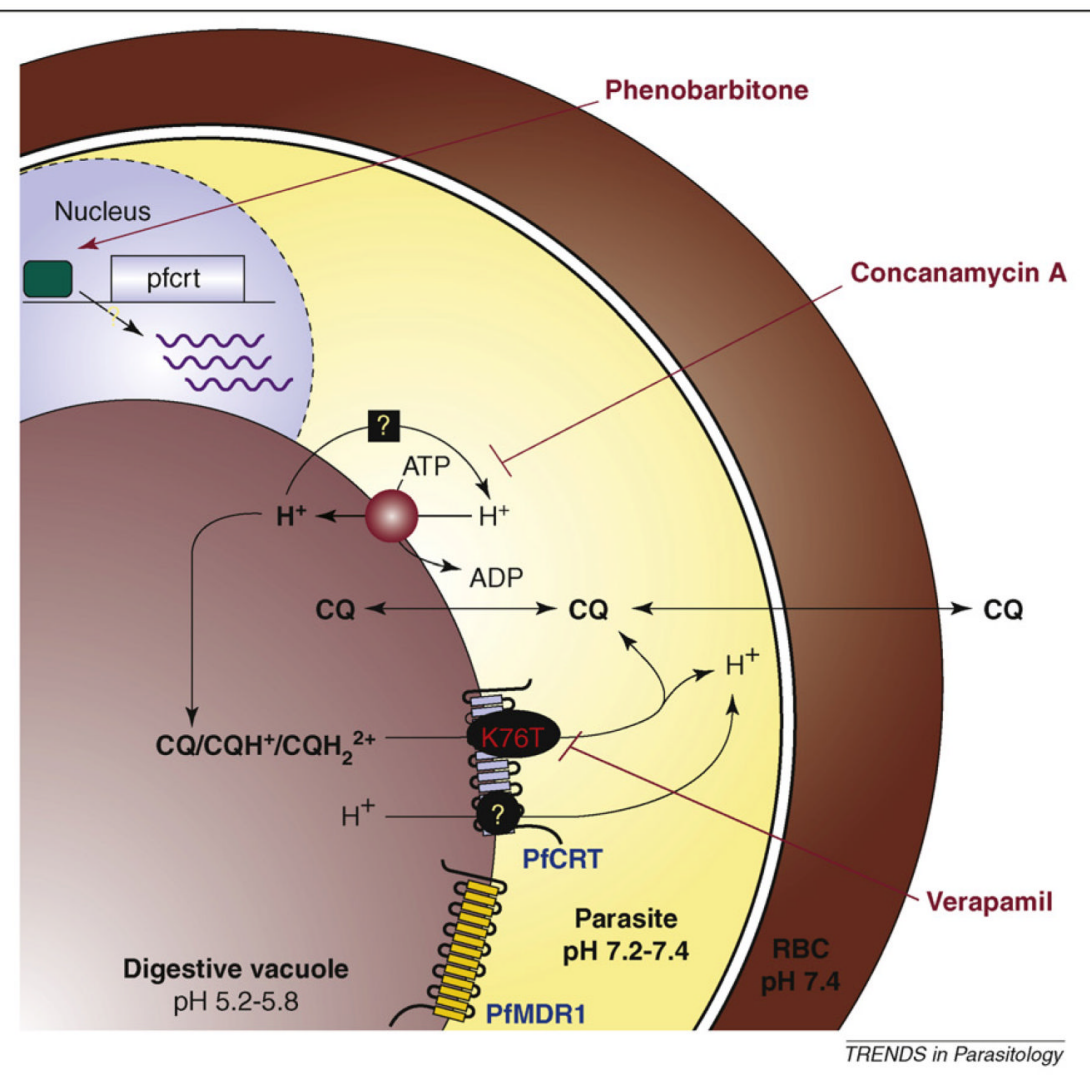

Figure 2.

Model of the chloroquine (CQ)-associated $\mathrm{H}^{+}$leak observed in CQ-resistant $P$. falciparum lines. The $\mathrm{pH}$ of the digestive vacuole (DV) reflects a balance between the inward $\mathrm{H}^{+}$pumping of the $\mathrm{V}$-type $\mathrm{H}^{+}$-ATPase and the outward leak of $\mathrm{H}^{+}$from the $\mathrm{DV}$, via as yet uncharacterized leak pathways (indicated by the boxed question mark). Upon inhibition of the V-type $\mathrm{H}^{+}-$ ATPase with concanamycin A, efflux of $\mathrm{H}^{+}$via the leak pathways results in DV alkalinization. The neutral form of CQ diffuses down a weak-base gradient and accumulates within the acidic environment of the DV. Therein, CQ becomes mostly diprotonated and, hence, less membranepermeant. In CQ-resistant parasites (indicated by K76T), mutant PfCRT-mediated efflux of protonated CQ might occur in symport with $\mathrm{H}^{+}$. Kirk and colleagues have hypothesized that this constitutes a verapamil-sensitive $\mathrm{H}^{+}$leak that is responsible for the CQ-associated increase in the rate of alkalinization after inhibition of the parasite V-type $\mathrm{H}^{+}$-ATPase [47]. The circled question mark denotes the possibility that the flux of $\mathrm{H}^{+}$via mutated PfCRT underlies the higher rate of alkalinization (after pump inhibition) seen in CQ-resistant parasites, as well as the possibility that the efflux of CQ via mutated PfCRT might be a $\mathrm{H}^{+}$-coupled (and, therefore, secondary active transport) process. The promoter regions of $p f c r t$ and $p f m d r l$ have also been proposed to contain phenobarbitone nuclear-receptor-binding sites, which suggests that nuclear-receptor-mediated responses to drug exposure might alter the regulation of these genes [53]. RBC, red blood cell. Adapted, with permission of the Company of Biologists, from Ref. [47]. 
Table 1

$P$. falciparum drug targets with active small molecule inhibitors currently under investigation

\begin{tabular}{|c|c|c|}
\hline Cellular localization & Target & Refs \\
\hline \multirow[t]{5}{*}{ Apicoplast } & DOXP reductoisomerase & [7] \\
\hline & Apicoplast gyrase & [8] \\
\hline & Apicoplast protein synthesis & [8] \\
\hline & Apicoplast transcription & [8] \\
\hline & Fatty acid synthesis & [76] \\
\hline \multirow[t]{12}{*}{ Cytosol } & Choline synthesis & [17] \\
\hline & Coenzyme A biosynthesis & [77] \\
\hline & Gamma-glutamylcysteine synthetase & [76] \\
\hline & Hypoxanthine-guanine phosphoribosyl transferase & [76] \\
\hline & Leucine aminopeptidase & [78] \\
\hline & Methionine aminopeptidases & [79] \\
\hline & Protein farnesyltransferase & [80] \\
\hline & Protein N-myristoyl transferase & [81] \\
\hline & Shikimate pathway & [76] \\
\hline & Thioredoxin reductase & [76] \\
\hline & Thymidylate synthase & [82] \\
\hline & Vitamin B6 synthesis & [83] \\
\hline Dense granules & Subtilisin-like proteases & [84] \\
\hline \multirow[t]{2}{*}{ Digestive vacuole } & Cysteine/aspartic proteases & [76] \\
\hline & Heme detoxification & [85] \\
\hline \multirow[t]{4}{*}{ Membrane } & Folate-biopterin transport & [86] \\
\hline & Hexose transport & {$[12,13]$} \\
\hline & V-type $\mathrm{H}^{+}$-ATPase & [14] \\
\hline & Host-parasite interactions during invasion & [26] \\
\hline \multirow[t]{2}{*}{ Mitochondria } & Dihydroorotate dehydrogenase & [21] \\
\hline & Mitochondrial bc1 complex & [23] \\
\hline \multirow[t]{4}{*}{ Nucleus } & Cyclin-dependent protein kinases (Pfmrk) & [76] \\
\hline & DNA & [87] \\
\hline & Histone acetyltransferase & [88] \\
\hline & Histone deacetylase & [5] \\
\hline Red blood cell & Heterotrimeric G protein & [89] \\
\hline
\end{tabular}

